specialization of the snail's brain that has been attributed to the appearance of the retractable nose. As Van $\mathrm{Mol}^{2}$ was the first to point out, a prominent procerebral lobe is present in all stylommatophore species but in no basommatophore species. Could the brain of caecilians have a comparable adaptation? The possibility is suggested by numerous analogies that are evident in the olfactory systems of snails and vertebrates. Convergent features include the cellular organization of the sensory epithelium, the presence of synaptic glomeruli and the generation of oscillating field potentials ${ }^{3,4}$. Given that snails and caecilians seem to be the only ani-

mals with mobile and retractible eye/nose structures, there might be curiosities to discover in the caecilian brain, especially in those regions that receive afference from the tentacle.

\title{
Ronald Chase
}

Department of Biology,

McGill University,

1205 Ave. Docteur Penfield,

Montreal, Quebec, H3A 1B1,

Canada

e-mail: rchase@bio1.lan.mcgill.ca

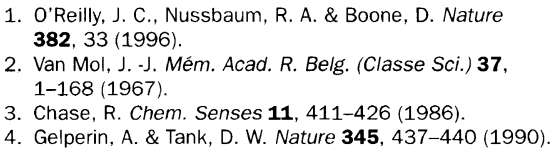

\section{Transposable element in fish}

SIR - Several transposable elements of the terminal-inverted-repeat class have been described in vertebrate genomes ${ }^{1}$. Some of them are thought to be active because of their presence in gene regions or polymorphic situations. However, demonstration of de novo excision or insertion of such elements has hitherto not been described to our knowledge. We have identified a novel terminal-invertedrepeat class transposable element in the medaka fish Oryzias latipes, and have detected its excision during embryogenesis.

The $i^{l} / i^{l}$ medaka fish genotype is associated with a complete albino phenotype ${ }^{2}$, as the $i$ allele is a defective tyrosinase gene $^{3}$. We isolated another allele at the same locus, $i^{4}$, from a commercial breed-

ing population: $i^{4} / i^{4}$ fish have a quasialbino phenotype. The $i^{4}$ allele is recessive to the wild-type allele $i^{+}$. A description of $i^{1}$ and $i^{4}$ is available on the World-Wide Web at the Medakafish home page (http://biol1.bio.nagoya-u.ac.jp: $8000 /$ ). Cloning and sequencing of the tyrosinase gene region of an $i^{4} / i^{4}$ fish reveals that the fifth exon contains a 4.7kilobase (kb) DNA insertion. We believe that the insertion sequence ( $a$ in the figure) is a terminal-inverted-repeat transposable element because: (1) it has inverted repeats at its termini; (2) there is duplication of a segment of the host chromosome; and (3) it is present as multiple copies. We have called this transposable element Tol2, and the particular copy found in the tyrosinase gene Tol2-tyr.
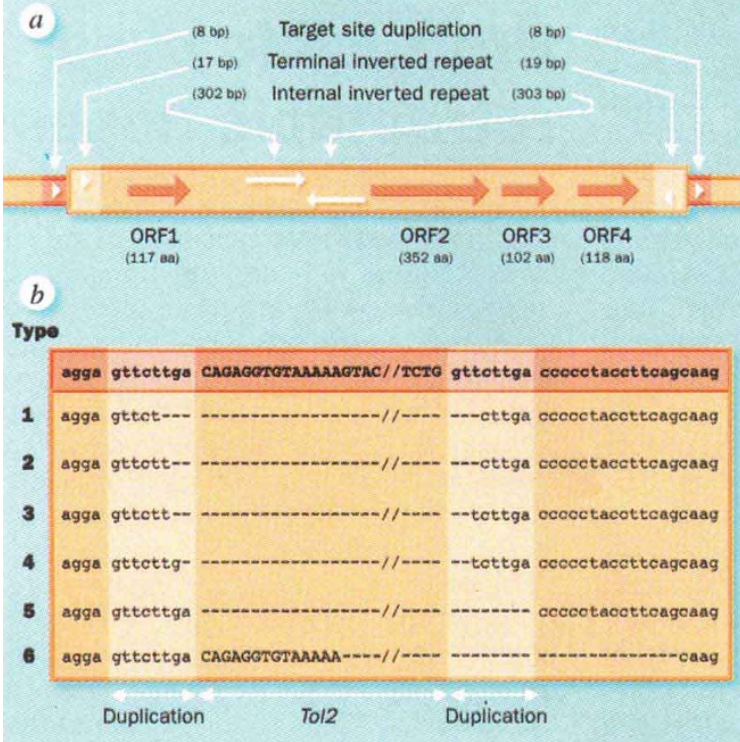

Characterization of To/2. a, Structure. Total length, 4,681 bp (GenBank accession number D84375). b. Excision footprints. Nucleotides of To/2 and the tyrosinase gene are shown in upper and lower case, respectively. Dash, no nucleotide occupation. The boxed sequence is from the genomic clone of the tyrosinase gene region that contains Tol2. About 10 copies are present in the diploid genome.

Tol2 contains 4 open

products. In addition, we observed $0.5-\mathrm{kb}$ fragments from 8 of the 60 embryos examined (see Supplementary Information). We cloned and sequenced these $0.5-\mathrm{kb}$ fragments and grouped the sequences obtained into six types, all featuring total, or neartotal, loss of the Tol2-tyr sequence ( $b$ in the figure). Type 5 is identical to the wild-type fish product. In the other five types, some nucleotides of the target site duplication or a Tol2 terminal region are retained. Thus our PCR analysis provides evidence for de novo excision of Tol2, with good but not absolute precision. Excision with some nucleotides left behind has also been observed for $A c^{9}$ and other terminal-inverted-repeat class transposable elements.

Whether Tol2-tyr itself is an autonomous member is not yet known. It is evident from our results, however, that an autonomous member is present somewhere in the genome. Tol2 is thus a unique tool for establishing a gene tagging system in fish, and possibly also in other vertebrate species.

\section{Akihiko Koga \\ Miho Suzuki \\ Hidehito Inagaki \\ Yoshitaka Bessho \\ Hiroshi Hori}

Department of Biology, Faculty of Science, Nagoya University, Nagoya 464-01, Japan

1. Plasterk, R. H. A. in Transposable Elements (eds Saedler, H. \& Gierl, A.) 125-143 (Springer, Berlin, 1996).

2. Tomita, H. in Medaka (Killifish): Biology and Strains (ed. Yamamoto, T.) 251-272 (Yugakusha, Tokyo, 1975).

3. Koga, A., Inagaki, H., Bessho, Y. \& Hori, H. Mol, Gen. Genet. 249, 400-405 (1995).

4. Fedoroff, N. V., Wessler, S. \& Shure, M. Cell 35 , 243-251 (1983).

5. Streck, R. D., MacGaffey, J. E. \& Beckendorf, S. K. EMBO J. 5, 3615-3623 (1992).

6. Sommer, H., Carpenter, R., Harrison, R. J. \& Saedler, H. Mol. Gen. Genet. 199, 225-231 (1985).

7. Atkinson, P. W., Warren, W. D. \& O'Brochta, D. A. Proc. Nati Acad. Sci. USA 90, 9693-9697 (1993).

8. Inagaki, H., Bessho, Y., Koga, A. \& Hori, H. Gene 150, 319-324 (1994).

9. Sutton, W. D., Gerlach, W. L., Schwartz, D. \& Peacock, W. J. Science 223, 1265-1268 (1984).

acid sequence similarity to $A c$ of maize ${ }^{4}$ hobo of Drosophila ${ }^{5}$ and Tam3 of snapdragon $^{6}$ (see Supplementary Information at Nature's website, http:/(www.nature.com). We propose that these elements have diverged from a single ancestor?.

Transposable elements of the terminal-inverted-repeat class are thought to move in a cut-and-paste fashion. To detect excision of Tol2 during embryogenesis, we conducted polymerase chain reaction (PCR) using a pair of primers which encompass Tol2-tyr and are 0.5-kb apart on the wild-type tyrosinase gene $^{8}$. We used genomic DNAs of 5-day-old albino embryos as templates. We examined 60 embryos individually, and all show $5.2-\mathrm{kb}$

\section{Dynamics of haemoglobin}

SIR - Jia et al. describe a possible physiological role of nitrosylated haemoglobin, postulating a dynamic cycle in which oxygenated haemoglobin in arterial blood is $S$-nitrosylated and the NO group is released to bind to the haem of deoxygenated haemoglobin during arterial-venous transit ${ }^{1}$. Thus the authors predict, citing ref. 2 as an example, that haemoglobin with haem-bound NO may not be observed in arterial blood but may be in venous blood. They contend also that the anticipated normal levels of haem-nitrosylated haemoglobin in vivo are too low to be detected by direct measurements (for example, electron paramagnetic resonance (EPR) spectroscopy). 